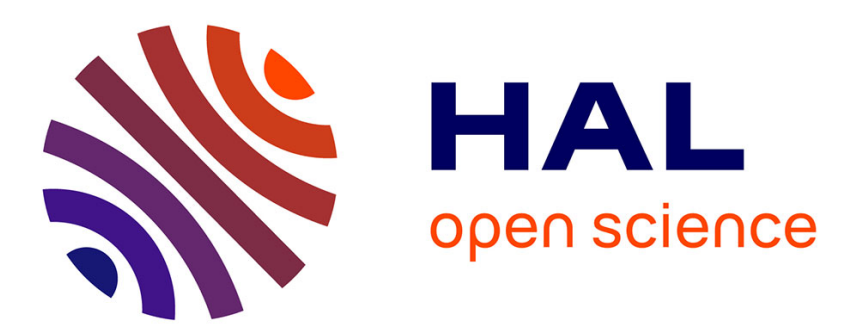

\title{
Dispersion compensation properties of grating-based temporal-correlation Optical Coherence Tomography systems
}

L. Froehly, L. Furfaro, P. Sandoz, P. Jeanningros

\section{To cite this version:}

L. Froehly, L. Furfaro, P. Sandoz, P. Jeanningros. Dispersion compensation properties of gratingbased temporal-correlation Optical Coherence Tomography systems. Optics Communications, 2009, 282 (7), pp.1488-1495. 10.1016/j.optcom.2008.12.038 . hal-00412316

\author{
HAL Id: hal-00412316 \\ https://hal.science/hal-00412316
}

Submitted on 12 May 2021

HAL is a multi-disciplinary open access archive for the deposit and dissemination of scientific research documents, whether they are published or not. The documents may come from teaching and research institutions in France or abroad, or from public or private research centers.
L'archive ouverte pluridisciplinaire HAL, est destinée au dépôt et à la diffusion de documents scientifiques de niveau recherche, publiés ou non, émanant des établissements d'enseignement et de recherche français ou étrangers, des laboratoires publics ou privés. 


\title{
Dispersion compensation properties of grating-based temporal-correlation Optical Coherence Tomography systems
}

\author{
L. Froehly*, L. Furfaro, P. Sandoz, P. Jeanningros \\ Département d'Optique PM Duffieux, Institut FEMTO-ST, UMR CNRS 6174, Université de Franche-Comté, 25030 Besançon Cedex, France
}

This article explores specific dispersion compensation properties of an OCT set-up based on temporal cor-relation performed by using a diffraction grating. The linear dispersion term introduced by a glass plate with a thickness of up to $9 \mathrm{~mm}$ has been experimentally compensated optically through the position adjustment of the output imaging lens and for a light bandwidth of $100 \mathrm{~nm}$. The dispersion compensation principle is described theoretically and results obtained experimentally are compared successfully with simulated ones. System limitations are discussed, especially regarding compensation of second orders of dispersion. Perspectives are given to achieve such a dispersion compensation.

\section{Introduction}

Optical Coherence Tomography is an ever growing technique for optical biopsies of tissues [1]. This echographic-like imaging modality shows an increased resolution with respect to its acoustic rival. Indeed the 3D resolution can reach the micrometer scale [2]. This resolution is directly related to the spectral bandwidth of the light source used for illumination. A simple way to increase the axial resolution is to increase the spectral bandwidth of the light source using, for example, supercontinuum light sources [3]. In fact dispersion is an unavoidable counterpart of this spectral broadening. Dispersion effects, due to material, optical components or sample under inspection, increase also with the spectral bandwidth. As a consequence of dispersion the axial resolution is decreased $[4,5]$.

The classical way to compensate for this dispersion is to introduce a compensation cell in the reference arm of the interferometer which primarily compensate the phase difference between interferometer arms due to differences in construction of the two arms and/or also due to variability of manufactured optical components. Then depending on the medium under inspection there could be a supplementary cell made of water, glass or different

\footnotetext{
* Corresponding author.

E-mail addresses: luc.froehly@univ-fcomte.fr (L. Froehly), luc.froehly@univfcomte.fr (L. Furfaro), luc.froehly@univ-fcomte.fr (P. Sandoz), luc.froehly@univfcomte.fr (P. Jeanningros).
}

materials depending on the medium under inspection. This supplementary cell thickness is chosen to be comparable with the mean depth of the tissue/media under inspection. A couple of prisms could then be added to compensate more accurately the residual dispersion which could be a residual dispersion of the system itself [2]. Of course compensation cells better have to be made of materials with dispersion properties close to those of the medium under inspection to compensate high order terms. This is especially true when speaking about Ultra High Resolution OCT systems [2]. In this case the effect of high order dispersion terms is increased.

Numerical dispersion compensation could also be realized at the cost of computation time [6]. Recently in Time Domain OCT (TDOCT), a delay line based on diffraction grating called RSOD (for Rapid Scanning Optical Delay line) was shown to be able to compensate for Group Velocity Dispersion [7-9]. The principle of this dispersion compensation relies on dispersion properties of diffraction grating already known for pulse compression (this is also the effect involved in the setup presented here). However TDOCT is also known to be slower than Fourier Domain OCT (FDOCT) with a sensitivity at least $20 \mathrm{~dB}$ lower [10]. It is also feasible to correct numerically for dispersion in Fourier space (FDOCT or Swept Source OCT) by a proper sampling of frequencies at the cost of computation time and/or more complex system (non linear sweeping of the laser wavelength) [11].

In the frame of this paper, we demonstrate interesting dispersion capabilities of an OCT system in which correlation is 
performed by means of a diffraction grating. To our knowledge, the first interferometric use of a diffraction grating for correlation purpose was reported in the field of spectroscopy [14] while it was applied to the measurement of phase objects (precursor of OCT) about one decade ago [15]. The principle as well as an exhaustive state of the art of grating-based temporal-correlation OCT can be found in $[12,13]$. The latter reference is a relevant background to this paper since it involves a set-up close to the one presented here. Therefore we briefly remind the principle of grating correlation in the following. In our configuration we demonstrate both theoretically and experimentally that the grating properties can be used for dispersion compensation purpose. We achieve the experimental compensation of a $9 \mathrm{~mm}$ thickness glass plate simply by shifting the imaging lens of the output detection system. We finally comment on limitations and future developments of this approach.

\section{System principle}

\subsection{System description}

The experimental set-up used is composed of three main parts as depicted in Fig. 1. Firstly the sample information is encoded via a Linnik interferometer. The latter is illuminated with a supercontinuum of light issued from a microstructured optical fiber pumped by a Q-switched Nd-YAG laser. The Supercontinuum has a full spectral range from $350 \mathrm{~nm}$ up to $1700 \mathrm{~nm}$ [16]. However in experiments reported here, the effective bandwidth is of $100 \mathrm{~nm}$ (Full Width at Half Maximum) centered around $650 \mathrm{~nm}$. Secondly the light beams issued from the Linnik interferometer are directed toward a second interferometer. In this Mach-Zehnder-like interferometer the output beam-splitter is replaced by a transmission diffraction grating disposed in the perpendicular direction. Because of the incident angles of the two beams, the transverse direction of the diffraction grating introduces a time-delay varying linearly between the recombined beams. The sample depth is thus encoded across the grating that forms a time correlation axis. The third part of the set-up is primarily an imaging system that forms the image of the diffraction grating on a two dimensional CCD image sensor ( 8 bits, $1256 \times 1024$ pixels, $15 \mathrm{fps}$ ). Then the lines of the CCD camera encode the depth of the sample and a A-scan is obtained without scanning along the image lines. The cascade of the two interferometers should result in autocorrelation. In practise, interfering beams are cross-polarized in both interferometers thanks to polarization multiplexing (quarter and half waveplates represented in Fig. 1). Thus we perform intercorrelation instead of autocorrelation.
Remark 1. We would like to emphasize that the correlation performed in the frame of this paper results from the interference of different beams than reported previously [13]. We used to consider a symmetrical configuration in which both beams issued from the Mach-Zehnder-like interferometer were diffracted in the -1 order by the grating before interfering. In the present case, a single beam considered for interference is diffracted in the -1 order. The other interfering beam is simply transmitted by the grating (This alternative was found to be more efficient for dispersion compensation as explained and shown in 2). Therefore the resulting expression of the output intensity takes a different form as described below.

\subsection{Theoretical background}

Basically the system could be viewed as a modified Young slit experiment (from spectral plane to detector plane D, see Fig. 1) where the distance between slits is wavelength dependent because of the grating law. The total recorded signal is then the sum in intensity of individual fringe patterns formed by each wavelength of the incident beams. Two temporal signals $r(t)$ and $s(t)$ with the same polarization state are incident onto the grating $(G)$ with opposite angles $\theta_{i}$ and $-\theta_{i} . r(t)$ and $s(t)$ have complex spectrum given by $\hat{R}(v)$ and $\hat{S}(v)$ respectively where $\hat{R}(v)$ and $\hat{S}(v)$ are Fourier transforms of $r(t)$ and $s(t) . \hat{R}(v)$ and $\hat{S}(v)$ can be expressed with their complex form $\hat{R}(v)=R(v) e^{j \cdot \varphi_{R}(v)}$ and $\hat{S}(v)=S(v) e^{j \cdot \varphi_{S}(v)}$. We consider in the following one diffracted beam ( -1 order) the other one being only transmitted by the diffraction grating (zero order). The modulus of the spectrum of the diffracted beam is directly accessible in the spectral plane of the spectrometer which is physically in the back focal plane of the lens L. The other beam that is simply transmitted by the grating is focused at a single point in the same back focal plane. Based on upper considerations a simple analysis leads to the expression of total intensity $C(z)$ in the plane D (the reader may refer to Ref. [13] since this analysis derives directly from this paper):

$C(z)=I_{0}+2 \mathscr{R} e\left[\int_{v} \hat{R}(v) \hat{S}(v) e^{-j 2 \pi\left(\frac{z}{\gamma c}\left(2 \sin \theta_{i}\right)\right) v} e^{j \frac{2 \pi z}{\gamma A}} d v\right]$

where $\mathrm{z}$ is the horizontal coordinate along the CCD camera lines, $I_{0}$ the background intensity, $\mathscr{R} e$ designs the real part. $\Lambda$ is the grating period, $\gamma$ the magnification of the imaging system. (The reference beam $\hat{R}(v)$ is assumed to be real while $\hat{S}(v)$ can be either real or complex depending on the optical sample properties). Eq. 1 shows clearly the correlation operation between the temporal fields realized by the system. The temporal variable is spatially displayed through the variable change.

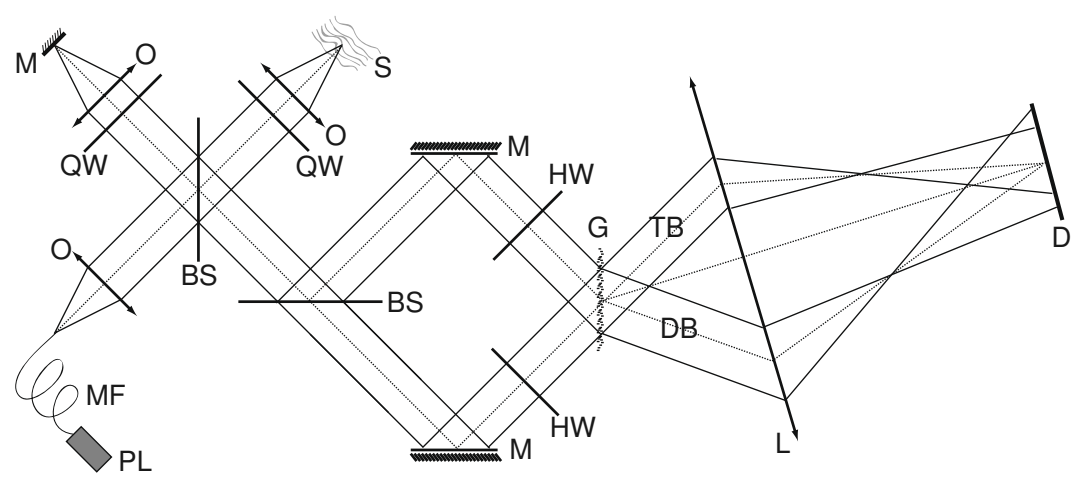

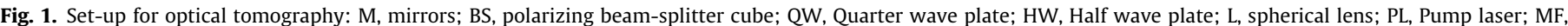

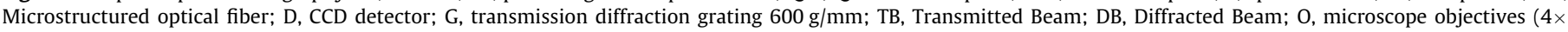
magnification); $\mathrm{S}$, sample. 


\section{Dispersion compensation using grating properties}

\subsection{Theory}

\subsubsection{General expression of the problem}

Fig. 2 shows the propagation of a plane wave of amplitude $a_{0}(\lambda)$, of wavelength $\lambda$, incident with an angle $\theta_{i}$ onto a grating $(\mathrm{G})$ with period $\Lambda$. The plane wave is diffracted and then propagates up to a plane $P$ parallel to the grating. $d$ is the distance between the grating and $P$. Let us now calculate the propagation of this plane wave from the grating plane to the observation plane $P$ at coordinate $z$ :

$a\left(z, d, \theta_{i}\right)=a_{0}(\lambda) e^{-j \frac{2 \pi}{\lambda} z \cdot \sin \theta_{d}} e^{-j \frac{2 \pi}{\lambda} d \cdot \cos \theta_{d}}$

with $\theta_{d}$ given by the grating law:

$\sin \theta_{d}-\sin \theta_{i}=-\frac{\lambda}{\Lambda}$

An other plane wave (non represented in Fig. 2) with amplitude $a_{p 0}(\lambda)$ is simultaneously incident with an angle $-\theta_{i}$ onto the grating. The transmitted beam (zero order) is superposed to the previous diffracted plane wave. This second plane wave after propagation through the observation plane $P$ expresses as:

$a_{p}\left(z, d, \theta_{i}\right)=a_{p 0}(\lambda) e^{j \frac{2 \pi}{i} z \cdot \sin \theta_{i}} e^{-j \frac{2 \pi}{i} d \cdot \cos \theta_{i}}$

then the total field in the plane $P$ at a distance $\mathrm{d}$ from the grating plane is:

$E\left(z, d, \theta_{i}\right)=a\left(z, d, \theta_{i}\right)+a_{p}\left(z, d, \theta_{i}\right)$

The resulting intensity is:

$$
\begin{aligned}
\left\|E\left(z, d, \theta_{i}\right)\right\|^{2}= & I+2 a_{0}(\lambda) \cdot a_{p 0}(\lambda) \cdot \cos \left[\frac{2 \pi}{\lambda} \cdot z\left(\sin \theta_{d}+\sin \theta_{i}\right)\right. \\
& \left.+\frac{2 \pi}{\lambda} \cdot\left(\cos \theta_{d}-\cos \theta_{i}\right) d\right]
\end{aligned}
$$

expressed now in function of the incidence angle $\theta_{i}$, Eq. 6 becomes:

$$
\begin{aligned}
\left\|E\left(z, d, \theta_{i}\right)\right\|^{2}= & I+2 a_{0}(\lambda) \cdot a_{p 0}(\lambda) \cdot \cos \left\{\left(\frac{4 \pi}{\lambda} z \cdot \sin \theta_{i}-\frac{2 \pi}{\Lambda} z\right)\right. \\
& \left.+\frac{2 \pi}{\lambda} \cdot\left[\sqrt{1-\left(-\frac{\lambda}{\Lambda}+\sin \theta_{i}\right)^{2}}-\cos \theta_{i}\right] \cdot d\right\}
\end{aligned}
$$

The phase of the fringe pattern results from the sum of two main terms:

$\Phi(z, d)=\left(\frac{4 \pi}{\lambda} \sin \theta_{i}-\frac{2 \pi}{\Lambda}\right) z+\frac{2 \pi}{\lambda} \cdot\left[\sqrt{1-\left(-\frac{\lambda}{\Lambda}+\sin \theta_{i}\right)^{2}}-\cos \theta_{i}\right] \cdot d$

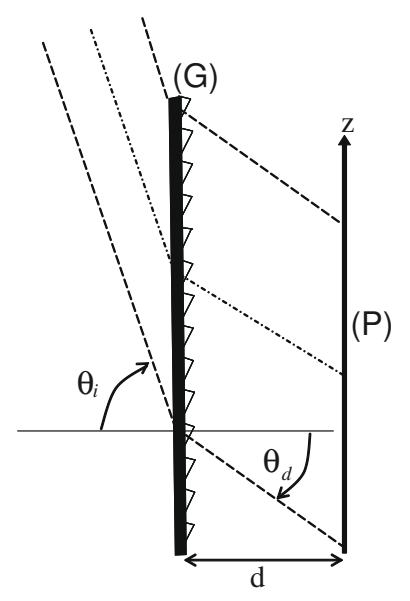

Fig. 2. Parameters for dispersion calculation.
The first term, proportional to $z:\left(\left[\frac{4 \pi}{2} \sin \theta_{i}-\frac{2 \pi}{4}\right] z\right)$, shows the classical interference pattern. It depends on the angle formed by the two plane waves and on the wavelength. The additional term $\left(\frac{2 \pi}{A} z\right)$ is responsible for a fringe frequency shift due to diffraction by the grating [13]. The second term, proportional to $d:\left(\frac{2 \pi}{\lambda}\left[\sqrt{1-\left(-\frac{\lambda}{\Lambda}+\sin \theta_{i}\right)^{2}}-\cos \theta_{i}\right] \cdot d\right)$, is non linear with light frequency but linear with $d$. This term is responsible for group velocity dispersion as well as higher order dispersion terms. We already saw in Eq. 8 that this dispersion can be either positive or negative depending on $d$.

Remark 2. At this stage we could explain our choice for this configuration with a single diffracted beam (remark in 2.1): in fact with the configuration described in [13], the resulting phase term is not sensitive to the defocusing $d$. Indeed the dispersion term for both diffracted arms is the same for equal $d$. The resulting phase dispersion terms compensate then. The only way to add a differential dispersion for this configuration is to induce a differential $d$ between the two diffracted beams. A tilt of the grating then breaks the symmetry and a differential $d$ results from this operation. Unfortunately this configuration needs a strong tilting of the grating to reach significant dispersion compensation.

We simulated the correction that is performed with a diffraction grating of 600 grooves $/ \mathrm{mm}$ enlightened under an incidence angle of $10^{\circ}$ with a distance $d=-2.5 \mathrm{~mm}$. Results show that it is possible to correct the dispersion of a $10 \mathrm{~mm}$ glass plate. The resulting path length difference is less than $3 \mu \mathrm{m}$ over a full spectral bandwidth of $100 \mathrm{~nm}$ (the equivalent coherence length is about $4 \mu \mathrm{m}$ ) to be compared to an initial dispersion larger than $30 \mu \mathrm{m}$ without correction. A more detailed discussion will be given in without correction. A more detailed discussion will be given in section 3.3.2.

\subsubsection{Generalization of the correlation formula}

Based on the previous paragraph analysis and on Eq. 1 we derive a general expression for the correlogram in the observation plane $P$ at a distance $d$ from the grating:

$C(z)=I_{0}+2 \mathscr{R} e\left[\int_{v} R(v) \hat{S}(v) e^{-j 2 \pi\left(\frac{z}{\gamma c}\left(2 \sin \theta_{i}\right)\right) v} e^{j \frac{2 \pi z}{\gamma \lambda}} e^{j \frac{j \pi}{\pi}\left(\sqrt{1-\left(-\frac{\lambda}{\lambda}+\sin \theta_{i}\right)^{2}}-\cos \theta_{i}\right) d} d v\right]$

where $\hat{S}(v)$ is complex and contains the dispersion properties of the medium under observation.

In practice, the distance $\mathrm{d}$ is chosen through the adjustment of the output imaging system of the setup. Basically, the lens L of Fig. 1 is supposed to form the image of the grating on the CCD camera. By shifting slightly the lens position, the object plane associated to the CCD camera can be moved aside from the grating as depicted in Fig. 3. The relationship between the distance $d$ and the lens shift $d^{\prime}$ is given by:

$d=\frac{-d^{\prime}\left(d^{\prime}-\overline{F^{\prime} D}-\overline{F G}\right)}{-d^{\prime}+\overline{F^{\prime} D}}$

In our case $d^{\prime}$ is very small (few millimeters) compared to imaging distances involved in Eq. 10 so that the relation becomes essentially linear:

$d=\frac{-d^{\prime}\left(\overline{F^{\prime} D}-\overline{F G}\right)}{\overline{F^{\prime} D}}$

In the next paragraph the simulation to be compared to experimental results is calculated using Eq. 11 together with a modified version of Eq. 8 (see section 3.2.1). 


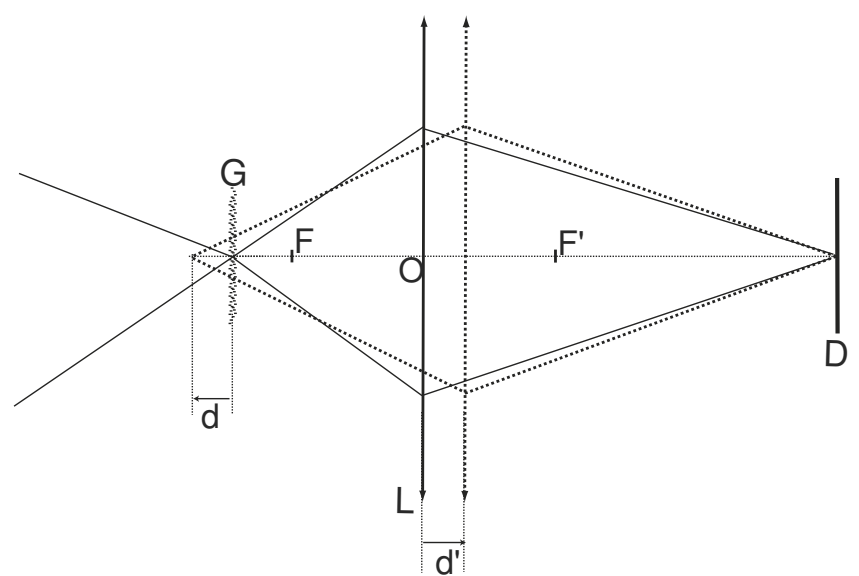

Fig. 3. Parameters for determining $d$ from the lens position $d^{\prime}$.

\subsection{Dispersion compensation measurements}

\subsubsection{Principle of measurements}

On Fig. 4 is depicted the experimental setup for realizing dispersion compensation. Only principles rays of beams are represented for a sake of clarity. We should first notice that the diffraction grat- ing is now represented rotated (as compared to Fig. 1) from an angle $\phi$ with respect to angle $\theta_{i}$ previously defined. Our choice for the discussion, in the first part, was to keep the grating symmetrical with respect to incidence angles to have a configuration more close to the one of [13] for a clarity reason. In practice, for current measurements the grating is in fact parallel to the detection plane. This will change slightly the phase expression given in Eq. 8. The new expression becomes:

$$
\begin{aligned}
\Phi(z, d)= & \left(\frac{4 \pi}{\lambda} \sin \theta_{i} \cdot \cos \phi-\frac{2 \pi}{\Lambda}\right) z \\
& +\frac{2 \pi}{\lambda} \cdot\left[\sqrt{1-\left(-\frac{\lambda}{\Lambda}+\sin \left(\theta_{i}-\phi\right)\right)^{2}}-\cos \left(\theta_{i}+\phi\right)\right] \cdot d
\end{aligned}
$$

This expression is now the basis for the following experiment: Glass plates (GP) are inserted in one arm of the interferometer (the MachZehnder-like one) and the lens L is shifted from a distance $d^{\prime}$ so that the dispersion induced by glass plates is balanced. We then calculate the theoretical compensation using Eq. 11 together with Eq. 12 for comparison with experimental results.

\subsubsection{Results}

Glass plates of thickness $1 \mathrm{~mm}$ are successively inserted (up to 9 plates) and for each plate inserted the position of the lens which

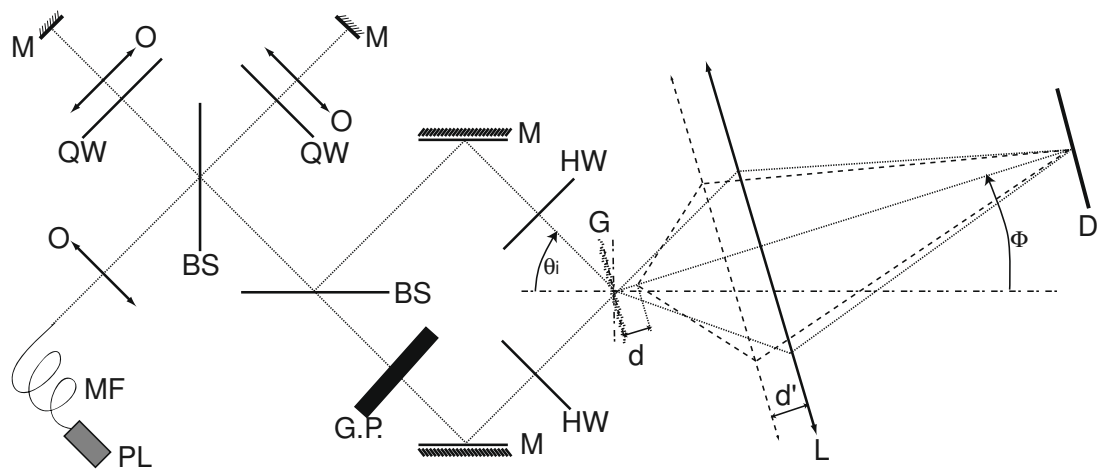

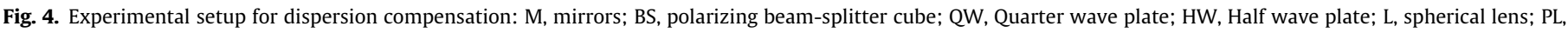
Pump laser; MF, Microstructured optical fiber; D, CCD detector; G, transmission diffraction grating; O, microscope objectives; GP, glass plates.

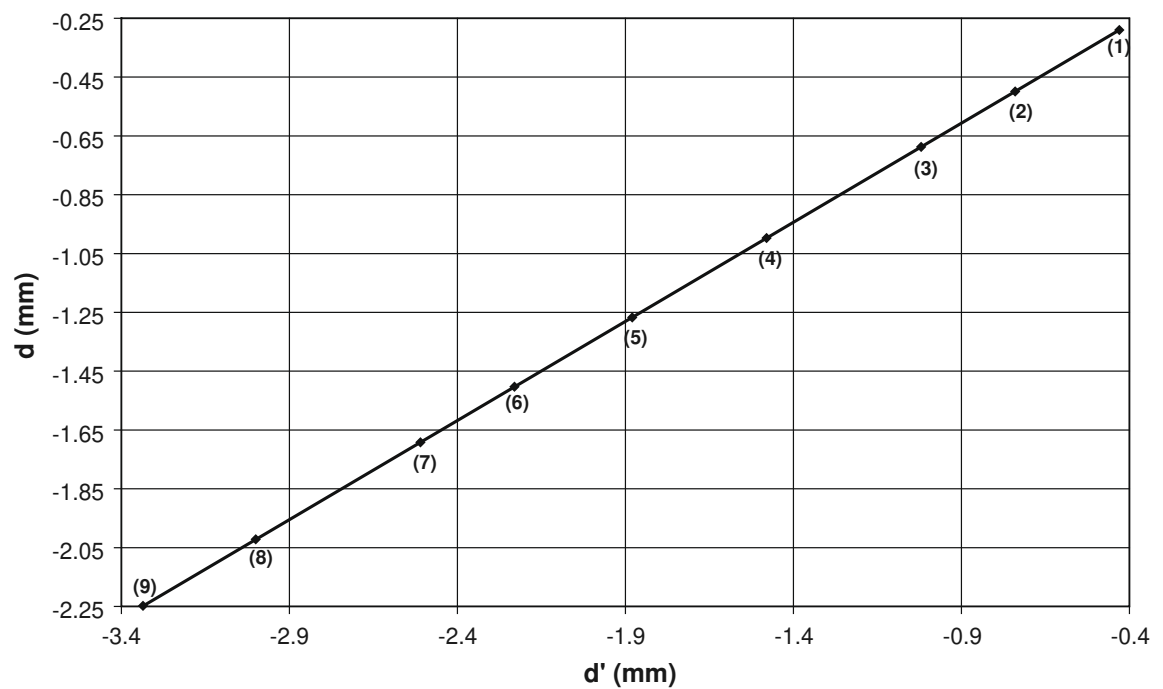

Fig. 5. Correspondence between $d^{\prime}$ and $d$ thanks to Eq. 11. Numbers labelled (1)-(9) are the number of glass plates inserted. 
allows dispersion correction is recorded. Fig. 5 gives the correspondence between the distances $d^{\prime}$ and $d$ as resulting from Eq. 11 as well as the set of lens positions permitting dispersion compensation after the insertion of 1 through 9 glass plates.

Fig. 6 is the output signal of the OCT system as recorded without any glass plate inserted; this is then the autocorrelation of the light source which will be the reference signal for dispersion compensation comparison. It appears on this figure that there are quite a few side lobs surrounding the central peak. This is essentially due to a band pass filter composed of a low pass filter together with a high pass filter (edge pass filters: Thorlabs-FKLP01-FKSP01) that we introduced in the set-up to extract a chosen bandwidth from the supercontinuum spectrum. This produces side lobes in the temporal space as a result of strong edges in the spectral plane (almost sinc function shape). The Figure gives a direct comparison between the autocorrelation measured and the autocorrelation obtained by Fourier transforming the measured power spectral density. The curve similarity observed validates the dispersion balancing of the system and that this recorded PSF constitutes an excellent reference for evaluating further dispersion compensation.

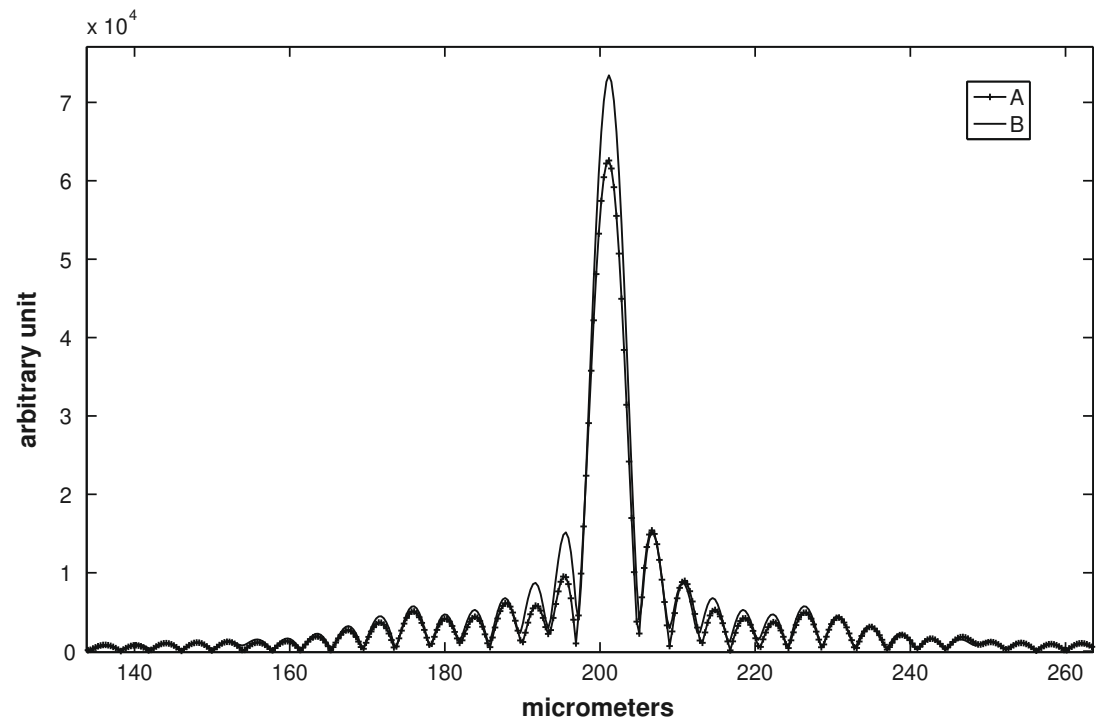

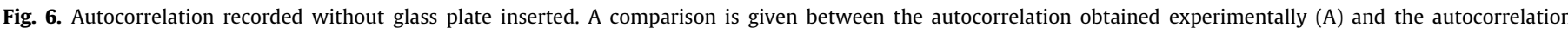
obtained by performing the Fourier transform of the measured power spectral density (B).

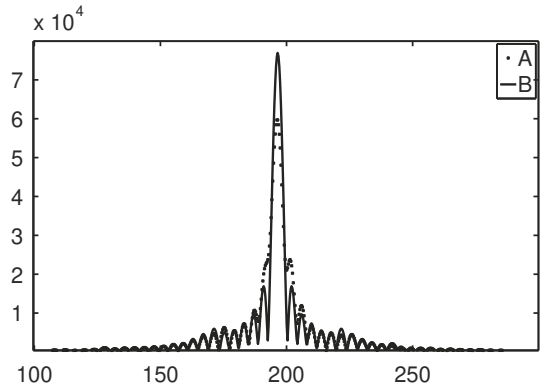

(a1)

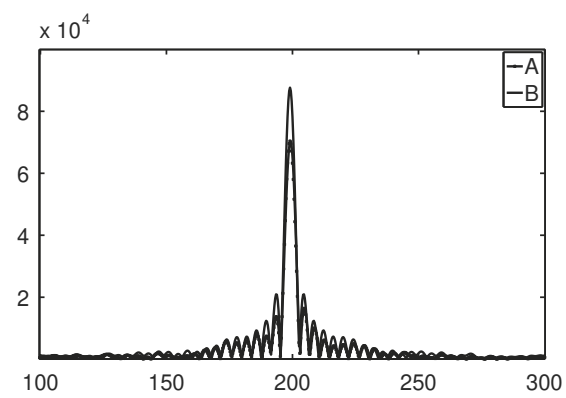

(a2)

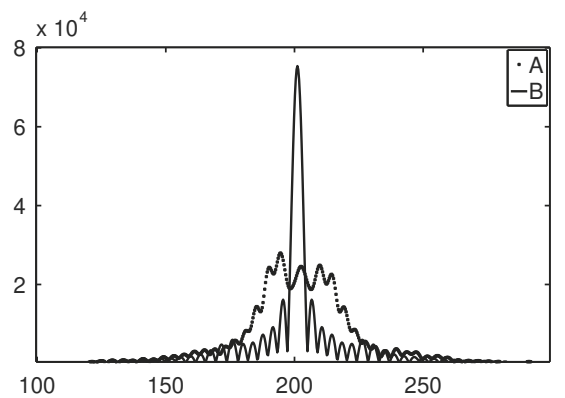

(b1)

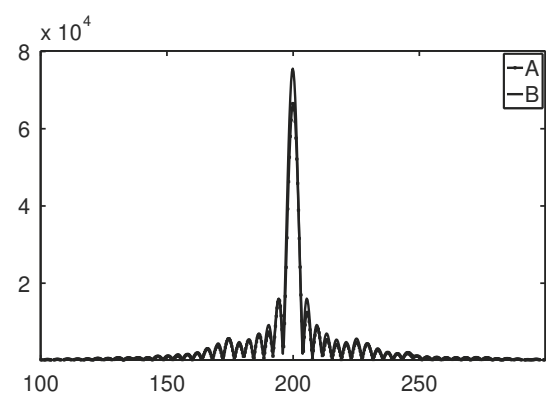

(b2)

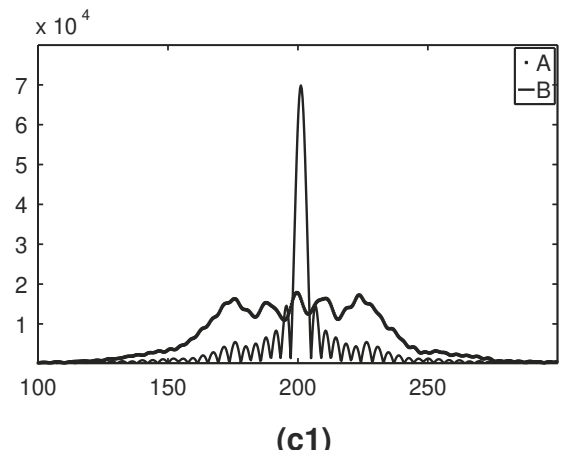

(c1)

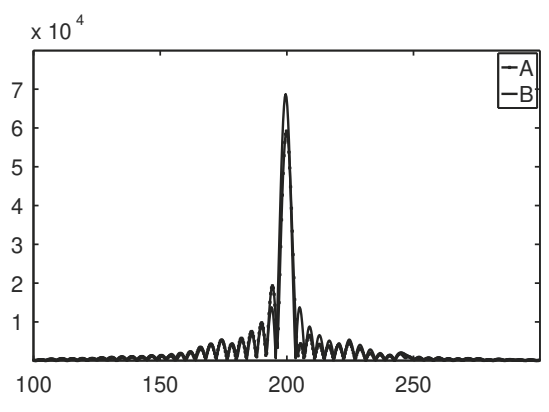

(c2)

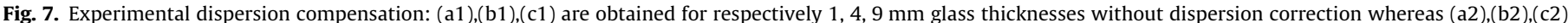

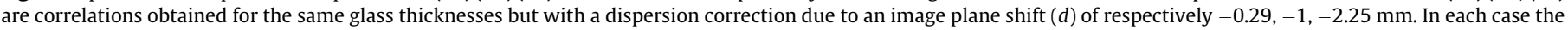
(A) curve is the autocorrelation that allows the comparison with the ideal Point Spread Function (PSF). 
Fig. 7 shows the recorded PSF with and without defocusing while different glass thicknesses are inserted in one arm of the interferometer. The excellent dispersion compensation is clearly seen and avoid any loss in both the signal magnitude or the effective coherence length (and therefore on the axial resolution). There is no visible sensitivity lost due to the defocusing since it remains pretty small. A sensitivity (and resolution also) lost would appear for higher optical frequencies since the defocusing would have to be increased too for compensating the same glass thicknesses. This effect is predicted by Eq. 12 through the non-linear dependency of dispersion with wavelength.

\subsection{Discussion}

\subsubsection{Comparison between theory and experiments}

Fig. 8 shows a comparison between experimental data and simulations based on Eq. 8. On this figure the defocusing $d$ necessary to obtain dispersion compensation is plotted versus the inserted glass thickness. It has to be noticed that theoretical values of $d$ have been calculated so that the residual dispersion (essentially a quadratic term) is lowered to its minimal value. There is a range of $d$ around this value where the PSF is almost unchanged as the residual dispersion remains narrower than the theoretical PSF (this range is represented through error bars). This explains the slight experimental deviations we notice in Fig. 8. In agreement with Fig. 7, we observe in Fig. 8 that all experimental values enter within the allowed d range that ensures a perfect PSF correction.

\subsubsection{Limitations and ways to pass them}

As already discussed in the section 3.3.2 the compensation obtained with the grating is excellent. Even with $9 \mathrm{~mm}$ of glass the resulting dispersion remains lower than $3 \mu \mathrm{m}$ over a $100 \mathrm{~nm}$ bandwidth. This is of the order of half the coherence length so that there is no PSF broadening as can be checked on Fig. 7. A closer look to the remaining dispersion term shows essentially a second order term (proportional to $v^{2}$ ). Fig. 9 shows the residual dispersion cal-

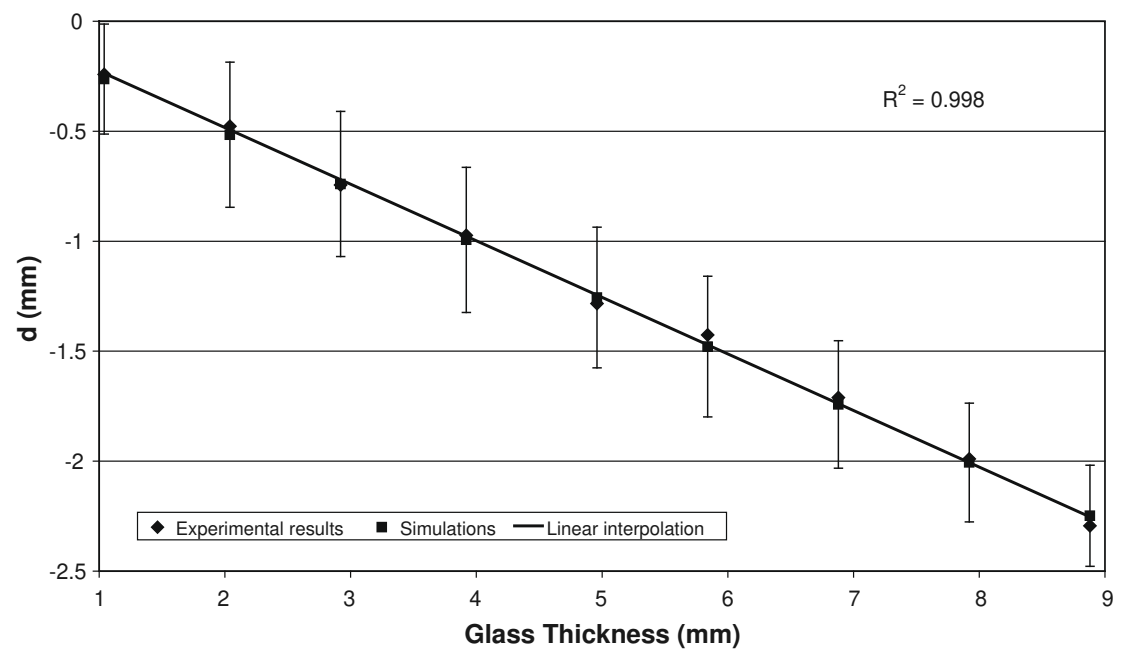

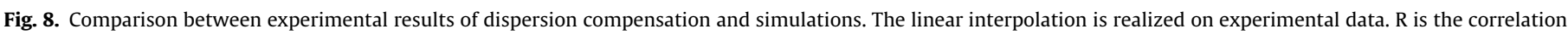
coefficient of this linear fit.

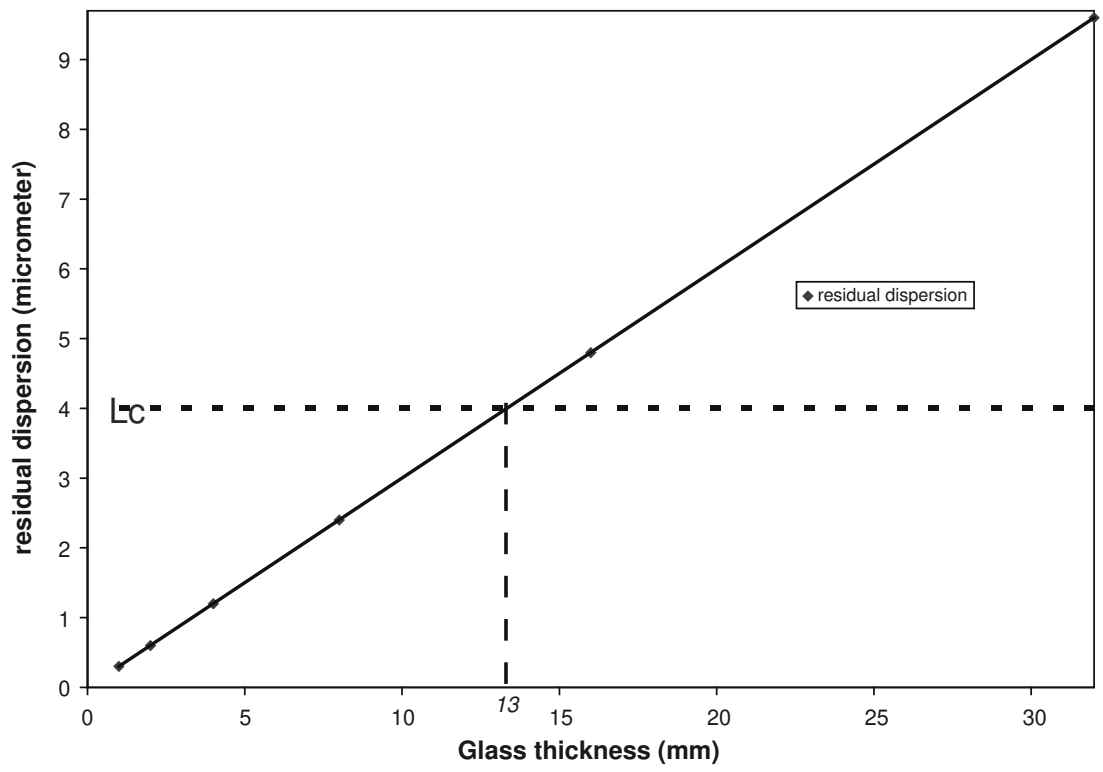

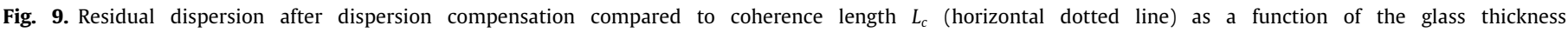
$\left(\lambda_{0}=650 \mathrm{~nm} ; \Delta \lambda=100 \mathrm{~nm}\right)$. 


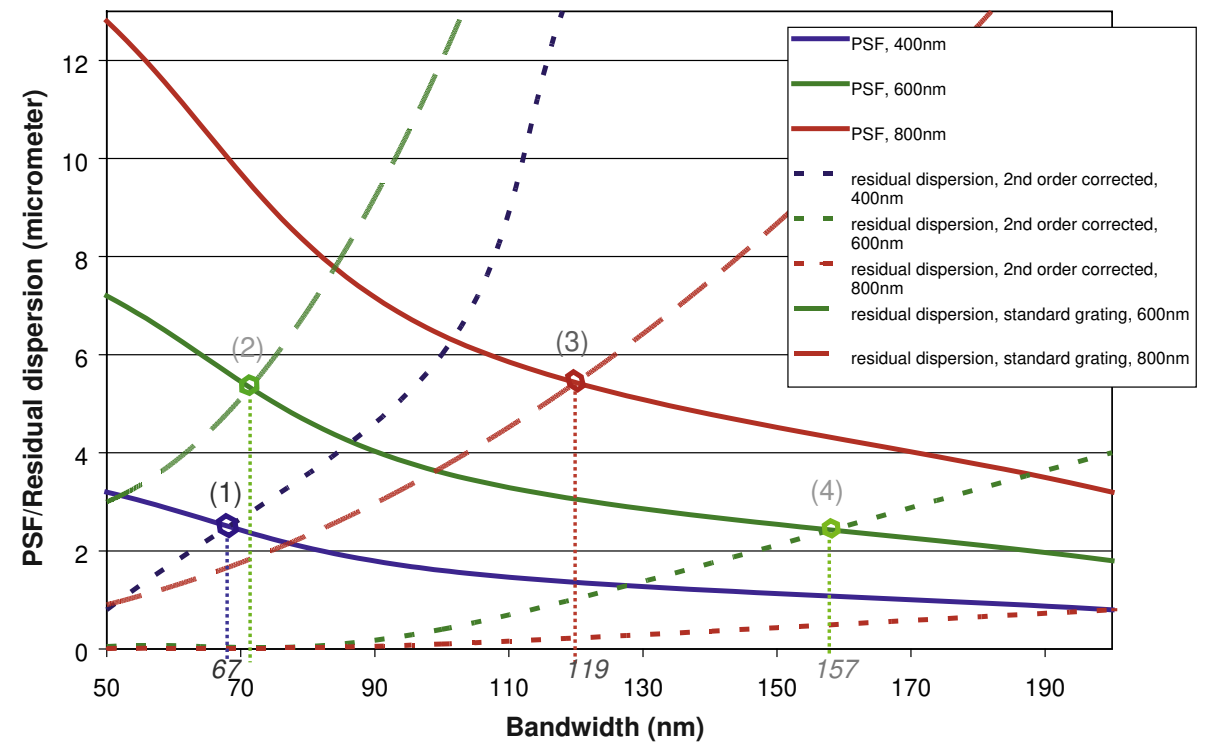

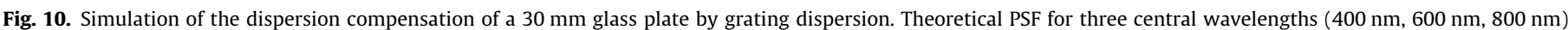

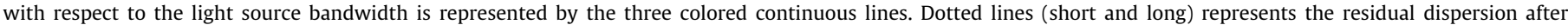

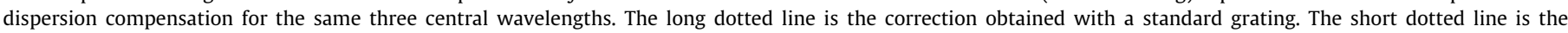

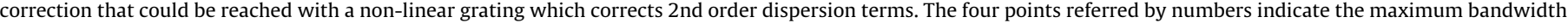
that can be compensated without PSF broadening for different $\lambda_{0}$ and dispersion terms compensated.

culated for glass thicknesses up to $32 \mathrm{~mm}$. The dotted line is the coherence length for a light source with a $100 \mathrm{~nm}$ bandwidth centered at $650 \mathrm{~nm}$. We can see than a PSF broadening appears only for glass thicknesses larger than $13 \mathrm{~mm}$. For thicker cases there is a coherence length lost due to second, third or higher orders of dispersion which are not compensated by defocusing. A simple solution to compensate thicker medium (as for example the eye) is to add a thick glass plate in the reference arm so that the differential thickness remains lower than $13 \mathrm{~mm}$. The residual dispersion could then be compensated finely by tuning the lens position.

Another issue which is out of the scope of this article would be based on non-constant period gratings to compensate for second order terms of the material dispersion. These gratings could indeed be designed to compensate exactly the dispersion of the system, including also the global dispersion of the observed medium. The fine tuning (for example due to the difference between eyes of several people) could then be realized by adjusting the lens as previously demonstrated. Fig. 10 shows a simulation (based on Eq. 12) of the dispersion compensation capability (a $30 \mathrm{~mm}$ glass thickness to compensate) when the first and second order of dispersion are now compensated (The grating period law needed to achieve such a compensation is out of the frame of this article). From this graph it appears clearly that the correction by a standard grating is easier in the red part or infra-red part of the spectrum. We see also that for ultra high resolution OCT it is necessary to compensate coarsely the dispersion of the system and the dispersion of the medium (if it is too thick) before using this kind of compensation for fine adjustment.

\section{Conclusion}

Dispersion properties of a grating-based OCT system have been presented and we have shown the possibility to compensate thick glass plates (as thick as $9 \mathrm{~mm}$ ) with a simple and easily tunable system. A slight axial displacement of the imaging lens acts as classical dispersion correction system composed of prism pairs. The perfect agreement between experimental and theoretical results has been demonstrated. The model developed and presented in this article allows the accurate prediction of the dispersion order correction efficiency for a specific diffraction grating, given light source parameters and setup geometry. Thanks to this model some future prospectives are proposed, as the possibility to perform non linear dispersion corrections by using chirped gratings. The chirp law could be computed knowing the dispersion law needed. A future work will be to design such chirp gratings to check how far we succeed in high order dispersion term correction. This is of special interest for Ultra High Resolution OCT. This article was also written to set the basic theory of dispersion compensation by grating-based correlation OCT systems. The scope of a forthcoming paper will be to show the capacity of this system to compensate for the depth dependant dispersion' of samples by simple optical means. Performances and limitations of such a system have to be demonstrated and analysed in comparison to other modalities cited previously $[8,11]$.

\section{Acknowledgements}

We acknowledge the French ANR for funding this work (ANR05-JCJC-0187-01) as well as our partners Leukos innovative solution and XLIM Institute in Limoges who optimized and provided us with compact supercontinuum light sources.

\section{References}

[1] A.F. Fercher, W. Drexler, C.K. Hitzenberger, T. Lasser, Rep. Prog. Phys. 66 (2003) 239.

[2] W. Drexler, U. Morgner, F.X. Kartner, C. Pitris, S.A. Boppart, X.D. Li, E.P. Ippen, J.G. Fujimoto, Opt. Lett. 24 (17) (1999) 1221.

[3] D. Reolon, M. Jacquot, I. Verrier, G. Brun, C. Veillas, Opt. Express 14 (1) (2005) 128.

[4] C.K. Hitzenberger, A. Baumgartner, A.F. Fercher, Opt. Commun. 154 (1998) 179

[5] C.K. Hitzenberger, A. Baumgartner, W. Drexler, A.F. Fercher, J. Biomed. Opt. 4 (1999) 144.

[6] D.L. Marks, A.L. Oldenburg, J.J. Reynolds, S.A. Boppart, Appl. Opt. 42 (2003) 204.

[7] G.J. Tearney, B.E. Bouma, J.G. Fujimoto, Opt. Lett. 22 (23) (1997) 1811.

[8] E.D.J. Smith, A.i V. Zvyagin, D.D. Sampson, Opt. Lett. 27 (22) (2002) 1998.

[9] W. Gao, Appl. Opt. 46 (6) (2007) 986. 
[10] R.A. Leitgeb, C.K. Hitzenberger, A.F. Fercher, Opt. Express 11 (2003) 889.

[11] A.R. Tumlinson, B. Hofer, A.M. Winkler, B. Povazay, W. Drexler, J.K. Barton, Appl. Opt. 45 (5) (2008) 687.

[12] L. Froehly, M. Ouadour, L. Furfaro, P. Sandoz, G. Huss, V. Couderc, Real-time optical coherence spectrotomography: proof of principle, in: SPIE (Ed.), Biophotonics and New Therapy Frontiers, vol. 6191, 2006.
[13] L. Froehly, M. Ouadour, L. Furfaro, P. Sandoz, G. Huss, V. Couderc, Int. J. Biom. Imag. 2008(Article ID 752340).

[14] P. Connes, J. Mod. Opt. 4 (1957) 136.

[15] I. Verrier, G. Brun, J.P. Goure, Appl. Opt. 36 (1997) 6225

[16] V. Tombelaine, C. Lesvigne, P. Leproux, L. Grossard, V. Couderc, J.L. Auguste, J.M. Blondy, G. Huss, P.H. Pioger, Opt. Express 13 (2005) 7399. 